\title{
The management of influenza and its complications within the Guy's and St Thomas' NHS Foundation Trust
}

\author{
Authors: Ahmed Hussain ${ }^{A}$ and Pratap Harbham ${ }^{B}$
}

\section{Background}

The peak number of admissions to hospital intensive care unit (ICU) for influenza-related complications was higher during the 2015/16 flu season than previous seasons. ${ }^{1}$ This coincided with a lower uptake of flu vaccination in targeted groups including the elderly, those clinically at risk and healthcare workers across the UK. ${ }^{1}$ This highlights the importance of optimal vaccination uptake in target risk groups.

\section{Aims}

We aimed to quantify vaccination uptake among over $65 \mathrm{~s}$ and clinically at risk groups as defined by Public Health England (PHE) in The Green Book. ${ }^{2}$ Furthermore, we wanted to evaluate current practice in the management of influenza against national guidelines and its complications, particularly community-acquired pneumonia (CAP) against local guidelines. $^{3,4}$

\section{Methodology}

Electronic records were interrogated for all influenza-positive ICU patients presenting to Guy's and St Thomas' NHS Foundation Trust (GSTT) between the 2015/16 and 2016/17 winter flu seasons. 97 patients were found to fit the inclusion criteria. Demographic, clinical, laboratory and outcome data were extracted using a standard data collection form. Chapter 19 of The Green Book defined clinically at-risk groups who should be offered annual flu vaccination. ${ }^{2}$ Antiviral drug selection was directly taken from national PHE 2016 guidance. $^{3}$ Local GSTT guidelines defined diagnostic and management standards for CAP. ${ }^{4}$

\section{Key findings}

> 69/97 (71\%) patients featured at least one co-morbidity, which qualified them to receive annual flu vaccination.

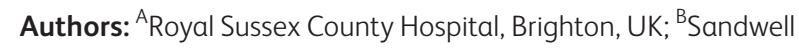
and West Birmingham NHS Trust, Birmingham, UK

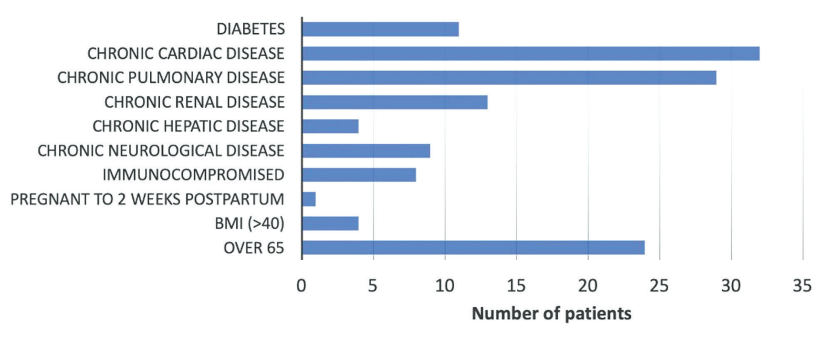

Fig 1. Presence of comorbidity which qualified patients to receive annual flu vaccination.

However, vaccination records were only documented in 4 (4\%) patients. This made evaluating vaccination uptake against national targets set by the Department of Health difficult (Fig 1)..$^{5}$

> 24/47 (51\%) H1N1 patients and 14/50 (28\%) non-H1N1 patients were not treated in compliance with national PHE guidance.

> 47/68 (69\%) patients with CAP were not given guideline antibiotic therapy; piperacillin/tazobactam was the most commonly misused antibiotic.

> 10/16 (63\%) of penicillin-allergic patients were either given the wrong antibiotic cover or a contraindicated antibiotic.

\section{Conclusion}

Current management of influenza and its complications remains inadequate. Increasing vaccination uptake in clinically at-risk groups remains a priority. Poor documentation of vaccination status makes evaluating vaccination status against national targets difficult. Lack of data hinders the development of targeted campaigns that could potentially improve uptake of flu vaccinations among target groups. Other actions include:

$>$ Mandatory screening of all patients presenting to hospital with influenza-type symptoms for flu vaccination records.

$>$ Require viral strain data to be sent, if available, with anti-viral drug requests, with the intention of restricting oseltamivir use to treat non-H1N1 influenza and zanamivir for H1N1 influenza.

> Arrange a meeting between the ICU pharmacists and infectious disease department to formalise the process of 
appropriate step-up/step-down of antibiotic regimes used in ICU for CAP based on CURB-65 score and formalise the use of piperacillin/tazobactam.

$>$ Weekly review of all patients with known drug allergies currently in ICU to be conducted by ICU pharmacists and/or infectious disease department.

\section{Conflicts of interest}

None declared.

\section{References}

1 Public Health England. Surveillance of influenza and other respiratory viruses in the United Kingdom: Winter 2015 to 2016. PHE, 2016.

2 Department of Health. Immunisation against infectious disease influenza - 'The Green book.' DOH, 2015.

3 Public Health England. PHE guidance on use of antiviral agents for the treatment and prophylaxis of seasonal influenza Version 7.0. PHE, 2016.

4 Guy's and St Thomas' NHS Foundation Trust. Clinical guideline: antibiotic use in adults. GSTT, 2015.

5 Public Health England. Flu plan: winter 2016 to 2017. PHE, 2016. 